\title{
Discussion of paper by C. E. Wagner and G. H. McKinley, entitled 'Age-dependent capillary thinning dynamics of physically-associated salivary mucin networks'
}

\author{
Citation: Journal of Rheology 61, 1327 (2017); \\ View online: https://doi.org/10.1122/1.5008882 \\ View Table of Contents: http://sor.scitation.org/toc/jor/61/6 \\ Published by the The Society of Rheology
}

\section{Articles you may be interested in}

Age-dependent capillary thinning dynamics of physically-associated salivary mucin networks Journal of Rheology 61, 1309 (2017); 10.1122/1.4997598

Preface: Special Issue on Associating Polymers Journal of Rheology 61, 1099 (2017); 10.1122/1.5008817

A new stochastic simulation for the rheology of telechelic associating polymers Journal of Rheology 61, 1293 (2017); 10.1122/1.4997592

Discussion of paper by G. W. Park and G. lanniruberto, entitled 'A new stochastic simulation for the rheology of telechelic associating polymers'

Journal of Rheology 61, 1307 (2017); 10.1122/1.5008881

Rheological properties of tough hydrogels based on an associating polymer with permanent and transient crosslinks: Effects of crosslinking density

Journal of Rheology 61, 1371 (2017); 10.1122/1.4997589

Discussion of paper by J. Zhao, K. Mayumi, C. Creton and T. Narita, entitled 'Rheological properties of tough hydrogels based on an associating polymer with permanent and transient crosslinks: Effects of crosslinking density'

Journal of Rheology 61, 1385 (2017); 10.1122/1.5008885

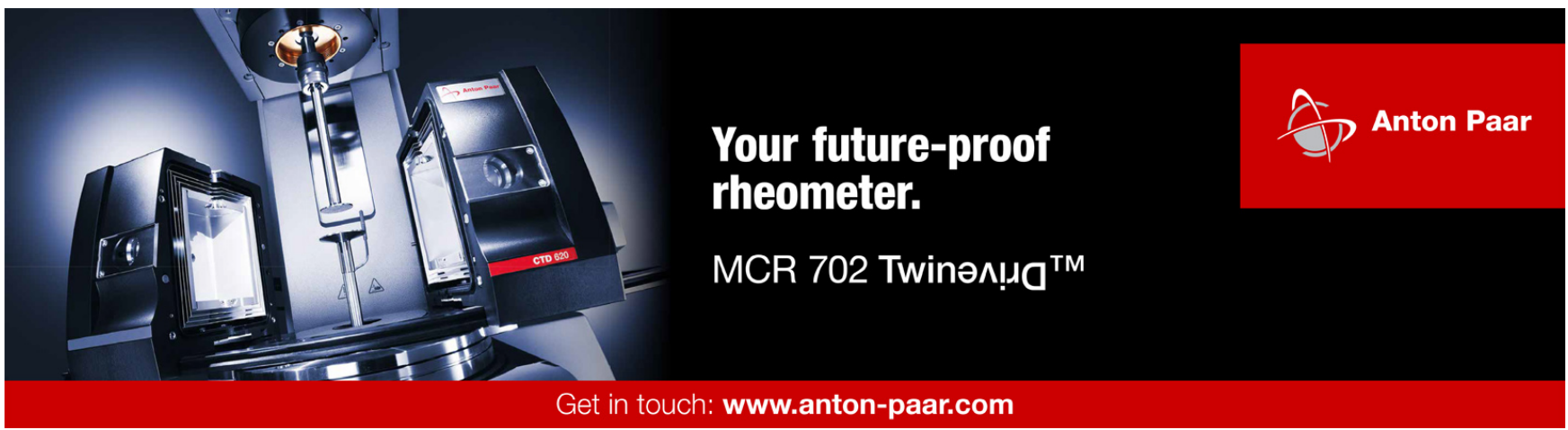




\title{
Discussion of paper by C. E. Wagner and G. H. McKinley, entitled 'Age-dependent capillary thinning dynamics of physically-associated salivary mucin networks'
}

\author{
(Received 10 October 2017; published 1 November 2017)
}

https://doi.org/10.1122/1.5008882

Jan Hendricks: Would a different prestretch (e.g., step height or strike time) influence the breakup behavior? I could imagine that the sample state and the amount of fully stretched or elastically active segments are altered.

Answer: Yes, we think that a different prestretch would absolutely affect the filament thinning dynamics and breakup behavior. This is also true for other viscoelastic materials tested in capillary breakup extensional rheometer (CaBER) because the magnitude of the initial strain affects the initial diameter of the sample (and thus the magnitude of the initial capillary pressure). For instance, under simple, uniaxial, extensional flow conditions, the sticky finitely extensible network (SFEN) model (as well as other models in the literature based on different constitutive equations) predicts an initial viscocapillary response prior to the onset of the well-known exponential filament thinning dynamics of the elastocapillary regime. In practice, however, particularly for low viscosity, water-based fluids (such as saliva), this viscocapillary response can terminate well before the experimental step duration (or "strike time"). We can only compare the predictions of the SFEN model with experimental data obtained at times greater than the strike time; and it is clear from the measurements that this initial rapid viscocapillary response is not observed in the experimental data despite it being a significant feature of the model response for the range of fluid parameters relevant for saliva. Various ways to address this discrepancy were investigated by Anna and McKinley [1] for polymer solutions of different solvent viscosities.

Our solution to this issue in the present paper is to initialize the mucin network with a small axial deformation in order to account for the stretch that would have been incurred during the separation of the top plate as well as in the short viscocapillary regime. The result of this is a nonzero polymer stress at $t=0$, which results in a much more rapid onset of elastocapillary thinning but also, as you correctly point out, a more rapid initial network junction dissociation rate. We suspect then that if the prestretch were substantially different such that the chains were much more (or much less) deformed prior to the strike time, it would likely be necessary to revise this initial condition, or better still to devise a more sophisticated way to account for a specific prestretch in the model arising from the initial step axial strain imposed by the plate separation.

Christian Clasen: In how far is the SFEN model capable of dealing with stronger stickers? Appendix D is an important addition to the paper, but the variation of the energy well depth in Fig. 8 is only $\pm 1 k T$. Although this probably covers the varying energy given in the literature for saliva, it would be of great interest to see how the model reacts to a variation by an order of magnitude (covering the relevant range for most other system presented in this JOR issue), if the breaking dynamics intrinsically changes with this variation, and in how far the model is still capable to capture experimentally observed thinning dynamics for stronger stickers.

Answer: In principle, the SFEN model is capable of accommodating arbitrarily strong stickers, even for $k T 1$ order of magnitude higher, as you propose. We selected the range shown in Appendix D to illustrate the sensitivity of the model response to small changes in parameter values of relevance to fitting a specific set of experimental data. Let's assume we follow the logic of the gedankenexperiment outlined in Appendix D, and hold $g \Omega$ constant while varying $\Delta G$. Then for very large values of $\Delta G$ (deep energy wells), the lifetime of network junctions $\lambda_{\text {exit }}$ becomes very large. Since the longest relaxation time extracted from CaBER measurements is linearly proportional to $\lambda_{\text {exit }}$ in the SFEN model [Eq. (30)], and during the elastocapillary regime the filament radius thins as $\exp \left(-t /\left(3 \lambda_{\text {exit }}\right)\right)$, then as $\lambda_{\text {exit }} \rightarrow \infty$ the model predicts that the filament radius remains constant. Therefore, all other parameters being equal (and chosen to be relevant for saliva), the SFEN model would not predict filament breakup (except at very long times) for values of the energy well depth that are 1 order of magnitude larger.

However, we suspect that other parameters of the model would also need to be adjusted in order to be physically representative of such a hypothetical fluid. For instance, the surface tension may be greater, which would result in stronger capillary forces. Furthermore, since $\lambda_{\text {exit }} \sim \exp (\Delta G / k T) / g$, a larger value of the scaling function $g$ (related to chain segment concentration and molecular weight) may also result in bringing the relaxation time back down and thus providing a reasonable/ finite filament thinning rate for a given value of $\Delta G$. Finally, if the finite extensibility of the chains was much smaller (or the energy well width much larger) such that the stickiness parameter $S k$ [Eq. (38)] was much larger and hence the network junction destruction rate diverged more rapidly, filament breakup may also be favored even for large values of $\Delta G$.

Evelyne van Ruymbeke: You nicely show that the thinning dynamics of the saliva strongly depends on the sample age, while the linear viscoelastic properties are nearly not affected by the time. This time evolution of elongation behavior is attributed to the decrease of the molar mass of the mucin molecules. Without knowing the system, I would have attributed these properties to a lower ability of the 
physical bonding to resist to flow with time (i.e., shorter lifetime of the physical bonds under stretch). Is this a plausible scenario?

Answer: This could certainly be an interesting explanation for the diminished elasticity observed in saliva with sample age. If this were the proposed mechanism, then it would be the parameter $\lambda_{\text {exit }}$ that would be reduced with age. This could come about from a smaller energy well depth $\Delta G$ or a larger value of $g$ (the scaling function related to chain segment concentration and molecular weight). Since the average molecular weight of the mucin chains in our model is larger than the molecular weight between sticky points at all ages and the concentration of mucin is not changing, it seems less likely that $g$ would be substantially altered. However, it is possible that the degradation of the mucin chains results in structural changes to the association sites that alter the number of moieties involved in the creation of a given cross-link, and so $\Delta G$ could possibly be reduced in this way. In addition, degradation and removal of the mucin sugar side chains could result in altered electrostatic repulsion profiles between polymers, which could also affect the strength of the other intermolecular bonds. Ultimately, since these phenomena are much more difficult to quantify and there is excellent evidence of the mucin chains diminishing in size with time due to enzymatic/proteolytic activity, we chose to incorporate temporal changes through the average molecular weight, although shorter junction lifetimes could also certainly play a role. This would however also change the shape of the filament thinning curves shown in Fig. 4; the rapid steep thinning in filament radius close to rupture is a distinctive hallmark of a highly extensible network with a few strong intermolecular bonds, rather than of a large number of weak bonds with low extensibility.
Evelyne van Ruymbeke: Also, the level of the plateau modulus in Fig. 5(a) is nearly constant, which means that the average molar mass of a sticky segment does not vary [see Eq. (1)], as well as the proportion of fast relaxing dangling ends. Therefore, can we attribute the strong thinning dynamics observed under extension to a weaker connectivity between the chains (i.e., each chain is associated with a lower number of other chains)?

Answer: Yes, the large degree of chain stretch under extension results in a large value of the ensemble average junction dissociation rate $M$ [Eq. (11)] and rapid filament thinning dynamics. In a sense then, since each mucin is composed of a certain number of sticky segments, the greater fraction of dangling ends at high values of $M$ corresponds to a given mucin molecule being less connected, on average, to other chains. In practice, however, the present SFEN model does not accurately account for this, since the higher order relaxation modes of entire mucin molecules with different degrees of connectivity (corresponding to different multisegment structures of different morphologies) are not considered. Instead, the sticky segments are treated more like individual units concatenated together to form a longer (and more extensible) macromonomer chain (which slowly decreases in length, or extensibility, with time). In principle, however, the evolution (and extensibility) of more complex connected microstructures and the resulting rheological characteristics could readily be numerically computed, as you have demonstrated in your own recent work.

\section{Reference}

[1] Anna, S., and G. McKinley, "Elasto-capillary thinning and breakup of model elastic liquids," J. Rheol. 45, 115-138 (2001). 